\title{
INFINITE SUBCLASSES OF RECURSIVELY ENUMERABLE CLASSES
}

\section{J. B. FLORENCE}

P. R. Young [1] has constructed an infinite recursively enumerable (r.e.) class with no proper infinite r.e. subclasses, and has asked if infinite r.e. classes with $m+1$ infinite r.e. subclasses exist for every $m \geqq 0$. It can further be asked what is the most general partially ordered set we can represent by the infinite r.e. subclasses of such a class (under inclusion). These questions are answered by the theorem below. The author wishes to thank A. H. Lachlan for his guidance and encouragement. Our construction is based on a formulation of Young's due to Lachlan.

Theorem. (a) Let $m \geqq 1, n \geqq 1$. Let $\left\{F_{i} \mid 1 \leqq i \leqq m+1\right\}$ be a class of subsets of $\{x \mid 1 \leqq x \leqq n\}$ closed to subsets and with

$$
\bigcup\left\{F_{i} \mid 1 \leqq i \leqq m+1\right\}=\{x \mid 1 \leqq x \leqq n\} .
$$

Then there is an infinite class $\mathfrak{C}^{*}$ of r.e. sets and distinct r.e. sets $A_{1}$, $\cdots, A_{n}$ not in $\mathrm{e}^{*}$ such that

$$
\mathfrak{C}=\mathfrak{C}^{*} \cup\left\{A_{i} \mid 1 \leqq i \leqq n\right\}
$$

is an infinite r.e. class with infinite r.e. subclasses

$$
\mathcal{e}-\left\{A_{i} \mid i \in F_{j}\right\} \quad(1 \leqq j \leqq m+1) .
$$

(b) There is an infinite r.e. class with one infinite r.e. subclass.

Conversely, any infinite r.e. class with finitely many infinite r.e. subclasses is of one of these forms.

Proof. We prove the converse first. Let $\mathfrak{e}$ be an infinite r.e. class with $m+1(m \geqq 1)$ infinite r.e. subclasses. If $\mathfrak{C}_{1}$ is an r.e. subclass and $X \in \mathfrak{e}-\mathfrak{e}_{1}$, then $\mathfrak{e}_{1} \cup\{X\}$ is an r.e. subclass. It follows that each infinite r.e. subclass lacks only finitely many members of $\mathfrak{e}$. Let $\mathfrak{e}^{*}$ be the intersection of the infinite r.e. subclasses, then $\mathfrak{C}-\mathfrak{C}^{*}$ is finite, with members $A_{1}, \cdots, A_{n}$, say $(n \geqq 1)$. Now the infinite r.e. subclasses of $\mathfrak{C}$ have the form

$$
\mathcal{e}-\left\{A_{i} \mid i \in F_{j}\right\} \quad(1 \leqq j \leqq m+1)
$$

where the $F_{j}$ are subsets of $\{x \mid 1 \leqq x \leqq n\}$. These are closed to subsets, for if $F \subseteq F_{j}$

Received by the editors June 13, 1966. 


$$
\mathcal{C}-\left\{A_{i} \mid i \in F\right\}=\left(\mathcal{C}-\left\{A_{i} \mid i \in F_{j}\right\}\right) \cup\left\{A_{i} \mid i \in F_{j}-F\right\}
$$

and the union of two r.e. classes is an r.e. class. Also, by definition of $A_{1}, \cdots, A_{n}$,

$$
\bigcup\left\{F_{i} \mid 1 \leqq i \leqq m+1\right\}=\{x \mid 1 \leqq x \leqq n\} .
$$

This completes the proof of the converse.

Note that in (a) $m+1 \leqq 2^{n}$. We give a construction for the case $m+1<2^{n}$ and obtain as corollaries the case $m+1=2^{n}$ and (b), both of which Young has already proved.

Since $m+1<2^{n}$ there are subsets $G_{1}, \cdots, G_{1}$ say of $\{x \mid 1 \leqq x \leqq n\}$ different from all the $F_{i}$. For each $i, k(1 \leqq i \leqq l, 1 \leqq k \leqq m+1)$ there is a number

$$
p(i, k) \in G_{i}-F_{k},
$$

since the $F_{i}$ are closed to subsets. For each $k$ with $1 \leqq k \leqq m+1$ there will be a different variation of the construction. We will show that these variations in the construction all give rise to the same $\mathfrak{C}^{*}$, $A_{1}, \cdots, A_{n}$.

At this point we make some informal remarks. $\mathcal{C}$ will be enumerated in an r.e. sequence

$$
\left\langle A_{1}, \cdots, A_{n}, V_{0}, V_{1}, \cdots\right\rangle .
$$

To begin with the sets in the sequence are all disjoint. By "amalgamating" sets we force the following situation: there will be an increasing function $r(i)$ such that the $V_{i}$ different from each of $A_{1}, \cdots$, $A_{n}$ are $V_{r(0)}, V_{r(1)}, \cdots$. These will be disjoint from each other and from each of $A_{1}, \cdots, A_{n}$. Also if an r.e. set $W$ intersects infinitely many of $V_{r^{\prime}(0)}, V_{r(1)}, \cdots$ it intersects them all and

$$
1 \leqq i \leqq l \Rightarrow(E x)\left[x \in W \&(z)\left(x \in A_{z} \Leftrightarrow z \in G_{i}\right)\right]
$$

Now if we take $W$ to be $U \mathcal{C}_{1}$ where $\mathfrak{C}_{1}$ is an infinite r.e. subclass we get for all $i V_{r(i)} \in \mathcal{C}_{1}$ and for all $i$ with $1 \leqq i \leqq 1$ there is $z \in G_{i}$ with $A_{z} \in \mathfrak{C}_{1}$. Thus the only possibilities for $\mathfrak{C}_{1}$ are

$$
\mathcal{e}-\left\{A_{z} \mid z \in F_{k}\right\} \quad(1 \leqq k \leqq m+1) .
$$

$A_{x}, r(x)$ and $V_{r(x)}$ will be independent of the variation used to get the sequence

$$
\left\langle A_{1}, \cdots, A_{n}, V_{0}, V_{1}, \cdots\right\rangle,
$$

but in variation $k$ use of the function $p$ will ensure for no $y, z$ do we have 


$$
V_{y}=A_{z} \quad \text { with } \quad z \in F_{k},
$$

and thus an enumeration of $\mathrm{e}-\left\{A_{z} \mid z \in F_{k}\right\}$ is easily obtained. The number $r(i)$ is approximated to by a sequence

$$
r(i, 0), r(i, 1), \cdots \text {. }
$$

Because we care only about r.e. sets $W$ which intersect $V_{r(j)}$ for infinitely many $j$ we are able to allow $W$ to make $r(i, s+1) \neq r(i, s)$ only if $i>e$ (the index of $W$ ) and thus to make the sequence constant eventually. Now we proceed with the details.

Variation $k$. Let $\left\langle W_{e} \mid e \geqq 0\right\rangle$ be an r.e. sequence enumerating all the r.e. sets. Let $P=\left\{(d, e) \mid d \in W_{e}\right\}$. The sth pair will mean the $s$ th member of $P$ to appear in an effective enumeration of $P$ without repetitions. We define:

$$
W_{e}^{s}=\{d \mid(d, e) \text { is among the first } s \text { pairs }\} .
$$

We define for each $s \geqq 0$, by induction on $s$, sets $A_{1}^{s}, \cdots, A_{n}^{s}$ and a sequence of sets $\left\langle V_{0}^{s}, V_{1}^{s}, \cdots\right\rangle$. Let

$$
A_{x}^{0}=\{x-1\} \quad(1 \leqq x \leqq n), \quad V_{x}^{0}=\{n+x\} \quad(x \geqq 0) .
$$

Assume for induction

(1) the $V_{x}^{s}, A_{x}^{s}$ are all finite;

(2) the $A_{x}^{s}$ are all different, in fact $x-1 \in A_{x}^{s}-U\left\{A_{y}^{s} \mid y \neq x\right\}$;

(3) only finitely many of the $V_{x}^{s}$ can be the same;

(4) if $V_{x}^{s}$ is different from all the $A_{y}^{s}$ then it is disjoint from them all;

(5) of the $V_{x}^{s}$ which differ from all the $A_{y}^{s}$, different ones are disjoint;

(6) $V_{0}^{s}$ is different from all the $A_{x}^{s}$, properties evidently possessed by the $A_{x}^{0}, V_{x}^{0}$.

We can define a function $r(i, s)$ by

$$
\begin{aligned}
& r(0, s)=0, \\
& r(i+1, s)=\mu x\left[x>r(i, s) \& V_{x}^{8} \neq A_{j}^{8} \text { for } 1 \leqq j \leqq n\right. \\
& \left.\& V_{x}^{8} \neq V_{r(j, s)}^{8} \text { for } 0 \leqq j \leqq i\right] .
\end{aligned}
$$

Suppose the $(s+1)$ th pair is $(d, e)$ and there is a $j>e$ such that $d \in V_{r(j, s)}^{s}$. There is at most one such $j$ by (5). Define

$$
R(e, s, i, x)=x \in W_{e}^{s+1} \&(z)\left(x \in A_{z}^{s} \Leftrightarrow z \in G_{i}\right) .
$$

Case 1 . There is $i$ with $1 \leqq i \leqq l$ and $\sim(E x) R(e, s, i, x)$. Let $i$ be the least such number. Put 


$$
\begin{aligned}
& A_{x}^{8+1}=A_{x}^{s} \cup V_{r(j, s)}^{s} \quad \text { if } x \in G_{i}, \\
& A_{x}^{s+1}=A_{x}^{s} \quad \text { if } x \notin G_{i} \text {, } \\
& V_{x}^{s+1}=A_{y}^{s+1} \quad \text { if } V_{x}^{s}=A_{y}^{s} \text { for some } y \text { (there can be only } \\
& \text { one such } y \text { by (2)), } \\
& V_{x}^{s+1}=A_{p(i, k)}^{s} \cup V_{r(j, s)}^{s} \quad \text { if } V_{x}^{s}=V_{r(j, s)}^{s}, \\
& V_{x}^{s+1}=V_{x}^{*} \quad \text { otherwise. }
\end{aligned}
$$

This is where variation $k$ arises.

Case 2. Case 1 does not obtain but there is $i$ with $i<j$ and $W_{e}^{s+1}$ $\cap V_{r(i, s)}^{s}=\varnothing$. Let $i$ be the least such number. Put

$$
\begin{aligned}
& A_{x}^{s+1}=A_{x}^{s} \quad \text { for all } x, \\
& V_{x}^{s+1}=V_{r(i, s)}^{s} \cup V_{r(j, s)}^{s} \quad \text { if } V_{x}^{s}=V_{r(i, s)}^{s} \text { or } V_{r(j, s)}^{s}, \\
& V_{x}^{s+1}=V_{x}^{s} \quad \text { otherwise. }
\end{aligned}
$$

If there is no $j>e$ such that $d \in V_{r(j, s)}^{s}$ or if neither Case 1 nor Case 2 occurs, put

$$
A_{x}^{s+1}=A_{x}^{s} \text { for all } x ; \quad V_{x}^{s+1}=V_{x}^{s} \text { for all } x .
$$

We show that (1), . , (6) are preserved. We have $A_{x}^{s} \subseteq A_{x}^{s+1}$, $V_{x}^{s} \subseteq V_{x}^{s+1}$ (so that the $A_{x}^{s}, V_{x}^{s}$ are all nonempty), $V_{x}^{s}=V_{y}^{s} \Rightarrow V_{x}^{s+1}=V_{y}^{s+1}$, $V_{x}^{s}=A_{y}^{s} \Rightarrow V_{x}^{s+1}=A_{y}^{s+1}$ for all $x, y$. (1) and (3) are clear.

For (2) we need consider only Case 1 , where the result follows by induction hypothesis (4) and the definition of $r$.

For (4) and (5), consider $Y_{x}^{s+1}, V_{y}^{s+1}$ different from each other and from all the $A_{z}^{s+1}$. Then $V_{x}^{s}, V_{y}^{s}$ are different from each other and from all the $A_{z}^{s}$, so by induction hypothesis (4), (5) they are disjoint from each other and from all the $A_{z}^{s}$. The desired conclusion is that $V_{x}^{s+1}$, $V_{y}^{s+1}$ are disjoint from each other and from all the $A_{z}^{s+1}$. If Case 1 occurs, then neither $V_{x}^{s}$ nor $V_{y}^{s}$ is equal to $V_{r(j, s)}^{s}$, for $V_{x}^{s}=V_{r(j, s)}^{s}$ implies that $V_{x}^{s+1}=A_{p(i, k)}^{s} \cup V_{\tau(j, s)}^{s}=A_{p(i, k)}^{s+1}$. Thus $V_{x}^{s+1}=V_{x}^{s} \quad$ and $V_{y}^{s+1}=V_{y}^{s}$. The two are therefore disjoint. Also $V_{x}^{s}$ is disjoint from $V_{r(j, s)}^{s}$ and from $A_{z}^{s}$ for all $z$, and so $V_{x}^{s+1}$ is disjoint from $A_{z}^{s+1}$ for all $z$. If Case 2 occurs at least one of $V_{x}^{s}, V_{y}^{s}$ is different from both $V_{r(i, s)}^{s}$ and $V_{r(j, s)}^{s}$, or $V_{x}^{s+1}=V_{y}^{s+1}$. If both have this property then $V_{x}^{s+1}=V_{x}^{s}$ and $V_{y}^{s+1}=V_{y}^{s}$ and the result follows since $A_{z}^{s+1}=A_{z}^{s}$ for all $z$. This leaves the case where say $V_{x}^{s}=V_{r(i, s)}^{s}$ and $V_{y}^{s}$ differs from both $V_{r(i, s)}^{s}$ and $V_{r(s, s)}^{s}$, then $V_{y}^{s+1}=V_{y}^{s}$ and the result follows by induction hypotheses (4), (5). 
(6) follows by a similar argument, using the fact that in the construction $j>0$.

Define:

$$
\begin{array}{ll}
A_{x}=\bigcup\left\{A_{x}^{s} \mid s \geqq 0\right\} & (1 \leqq x \leqq n), \\
V_{x}=\bigcup\left\{V_{x}^{s} \mid s \geqq 0\right\} & (x \geqq 0) .
\end{array}
$$

We can find the members of $A_{x}^{s}, V_{x}^{s}$ effectively from $x, s$ so

$$
\left\langle A_{1}, \cdots, A_{n}, V_{0}, V_{1}, \cdots\right\rangle
$$

is an r.e. sequence enumerating an r.e. class $\mathcal{e}$. Let $\mathfrak{e}^{*}$ $=\mathfrak{e}-\left\{A_{1}, \cdots, A_{n}\right\}$.

For each $x$ and all sufficiently large (s.l.) s,

$$
r(x, s) \text { is a constant, say } r(x) \text {. }
$$

We prove this by induction on $x . r(0, s)=0$ for all $s$, so $r(0)=0$. We suppose the result holds for all $y \leqq x$ and we show it holds for $x+1$. There is an $s_{0}$ such that if $s \geqq s_{0} r(y, s)=r(y)$ for all $y \leqq x$. In Case 1 or Case 2

$$
\begin{aligned}
& r(z, s+1)=r(z, s) \quad \text { if } z<j, \\
& r(z, s+1)=r(z+1, s) \quad \text { if } z \geqq j,
\end{aligned}
$$

for if we divide the members of the sequence

$$
V_{0}^{8}, V_{1}^{s}, \cdots
$$

which differ from each of $A_{1}^{s}, \cdots, A_{n}^{s}$ into equivalence classes under set equality, $V_{r(z, s)}^{s}$ is the first member of the $(z+1)$ th such class, and by (4) and (5) the only effect of either case on the computation of $r$ is the loss of the original $(j+1)$ th class. So if $r(x+1, s+1) \neq r(x+1, s)$ with $s \geqq s_{0}$, Case 1 or Case 2 occurs with $x+1 \geqq j>e$ and by our induction hypothesis $x+1=j$. If Case 1 occurs we have $R(e, t, i, d)$ for all $t>s$ by (4), because $d$ does not belong to $V_{r(j, t)}^{t}$ for any $j$. Now $r(x+1, s+1) \neq r(x+1, s)$ can hold for only finitely many $s \geqq s_{0}$ through Case 1 -at most $l$ times for each $e<x+1$, and through Case 2 -at most $x+1$ times for each $e<x+1$ by our induction hypothesis. Thus $r(x+1, s)=r(x+1)$, a constant for all s.l. $s$.

By (2) $A_{1}^{s}, \cdots, A_{n}^{s}$ are distinguished by the numbers $0, \cdots, n-1$ for all $s$, so $A_{1}, \cdots, A_{n}$ are.

We now wish to prove that the $V_{r(u)}$ are distinct, disjoint from each other and from all the $A_{u}$. Suppose $x \in V_{r(u)} \cap V_{r(v)}$ with $u \neq v$. Then for all s.l. $s x \in V_{r(u)}^{s} \cap V_{r(0)}^{s}$, so all s.l. $s, x \in V_{r(u, s)}^{s} \cap V_{r(0, s)}^{s}$, contradict- 
ing (5). Similarly $x \in V_{r(u)} \cap A_{v}$ contradicts (4). The $V_{r(u)}$ are therefore distinct since they are nonempty.

Now we show that for all $x$, either $V_{x}=V_{r(u)}$ for some $u$ or $V_{x}$ $=A_{p(u, k)}$ for some $u$. It follows by induction on $s$ that if $V_{x}^{s}$ is equal to one of the $A_{y}^{s}$ then it is equal to $A_{p(u, k)}^{s}$ for some $u$. Suppose that there is no $u$ such that $V_{x}=V_{r(u)}$. There is then a $u$ such that $r(u)<x$ $<r(u+1)$. Consider $s$ s.l. that $r(v, s)=r(v)$ for all $v \leqq u+1$. Then $V_{x}^{s}=A_{p(z, k)}^{s}$ for some $z$, in which case $V_{x}=A_{p(z, k)}$, or $V_{x}^{s}=V_{r(0, s)}^{s}$ for some $v \leqq u$, in which case $V_{x}=V_{r(v)}$.

By induction on $s, A_{x}^{s}, r(x, s)$ and $V_{r(x, s)}^{s}$ are all independent of $k$. Thus $A_{x}, r(x)$ and $V_{r(x)}$ are all independent of $k$.

Now we show that if $W_{e}$ intersects infinitely many of $V_{r(0)}$, $V_{r(1)}, \cdots$, then

$$
\begin{gathered}
1 \leqq i \leqq l \Rightarrow(E x) R(e, i, x), \text { where we define } \\
R(e, i, x)=x \in W_{\bullet} \&(z)\left(x \in A_{z} \Leftrightarrow z \in G_{i}\right) ; \\
i \geqq 0 \Rightarrow(E x)\left[x \in W_{\bullet} \cap V_{r(i)}\right] .
\end{gathered}
$$

First we have: if $a, t$ are any given numbers there is $s>t$ and $j>a$ such that the $(s+1)$ th pair is $(d, e)$ and $d \in V_{r(j, s)}^{s}$. For there are infinitely many $y>a$ such that $W_{e}$ intersects $V_{r(y)}$. Also these $V_{r(y)}$ are disjoint. So for infinitely many members $d$ of $W_{e}, d \in V_{r(y)}$ for some $y>a$. So there is $s>t$ such that $(d, e)$ is the $(s+1)$ th pair and $d \in V_{r(v)}$ with $y>a$. Now $d \in V_{r(1, s)}^{s}$ for some $j$. Suppose $j<y$. Consider $u>s$ s.l. that $r(y, u)=r(y)$ and $d \in V_{r(v)}^{u}$. Then $d \in V_{r(y, u)}^{u} \cap V_{r(j, s)}^{u}$ so by (4) and (5) $V_{r(v, u)}^{u}=V_{r(j, s)}^{u}$, but $r(y, u)>r(j, u) \geqq r(j, s)$ contradicting the definition of $r(y, u)$. Thus $j \geqq y \geqq a$.

Now suppose there is a least $i \leqq l$ such that $\sim(E x) R(e, i, x)$. For each $y<i$ let $d(y)$ be such that $R(e, y, d(y))$. Let $t$ be s.l. that for each $y<i R(e, t, y, d(y))$. Put $a=e$ and let $s, j$ correspond to $a, t$ as above. Since for each $y<i$ we have $R(e, s, y, d(y))$ and $\sim(E x) R(e, s, i, x)$ Case 1 ensures that $R(e, s+1, i, d)$ and so $R(e, i, d)$, contradiction.

Suppose there is a least $i$ such that $\sim(E x)\left[x \in W_{e} \cap V_{r(i)}\right]$. Let $t$ be s.l. that $r(i, t)=r(i)$, for each $y<i(E x)\left[x \in W_{e}^{t+1} \cap V_{r(y, t)}^{s}\right]$ and $r(y, t)=r(y)$ and for $1 \leqq y \leqq l R(e, t, y, d(y))(d(y)$ as above). Put $a=\max (e, i)$ and let $s, j$ correspond to $a, t$ as above. Then Case 2 ensures that $d \in W_{e}^{s+2} \cap V_{r(i, s+1)}^{s+1}$ so $(E x)\left[x \in W_{e} \cap V_{r(i)}\right]$, contradiction.

Recall that $\mathfrak{C}$ has distinct members

$$
A_{1}, \cdots, A_{n}, V_{r(0)}, V_{r(1)}, \cdots
$$

and $\mathfrak{e}^{*}=\left\{V_{r(0)}, V_{r(1)}, \cdots\right\}$. Suppose $\mathfrak{e}_{1}$ is an infinite r.e. subclass. Put $U \mathcal{C}_{1}=W_{\theta}$ (it is an r.e. set). $W_{0}$ intersects infinitely many of the 
$V_{r(i)}$ so we can apply (7) and (8). By (8), since the $V_{r(i)}$ are disjoint from each other and from all the $A_{i}, V_{r(i)} \in \mathrm{e}_{1}$ for all $i$. So the only possibilities for $\mathfrak{C}_{1}$ are

$$
\begin{array}{ll}
\mathcal{C}-\left\{A_{z} \mid z \in G_{i}\right\} & (1 \leqq i \leqq l) \\
\mathcal{C}-\left\{A_{z} \mid z \in F_{k}\right\} & (1 \leqq k \leqq m+1) .
\end{array}
$$

We discount the first possibility. For by (7),

$$
x \in W_{e} \&(z)\left(x \in A_{z} \Leftrightarrow z \in G_{i}\right) \quad \text { for some } x .
$$

$x \notin V_{r(j)}$ for any $j$ so the only members of $\mathfrak{e}$ which $x$ belongs to are the $\left\{A_{z} \mid z \in G_{i}\right\}$. So one of these sets must be in $\mathfrak{C}_{1}$.

We complete the proof by showing that

$$
\text { e }-\left\{A_{z} \mid z \in F_{k}\right\} \text { is r.e. } \quad(1 \leqq i \leqq m+1) .
$$

For consider the construction of $\mathfrak{e}$ by variation $k$, in an r.e. sequence

$$
\left\langle A_{1}, \cdots, A_{n}, V_{0}, V_{1}, \cdots\right\rangle \text {. }
$$

Since $V_{x} \neq$ any member of $\mathfrak{e}^{*} \Rightarrow V_{x}=A_{p(i, k)}$ some $i$ and $p(i, k) \notin F_{k}$, the r.e. sequence obtained by omitting the $z$ th member of the original one for each $z \in F_{k}$ enumerates the desired class.

(a) when $m+1=2^{n}$ : Let $n^{\prime}=n+1, m^{\prime}=2^{n+1}-2$ and the $F_{k}^{\prime}$ $\left(1 \leqq k \leqq 2^{n+1}-1\right)$ be all the subsets of $\{x \mid 1 \leqq x \leqq n+1\}$ other than the whole set. Let $\mathcal{C}, \mathfrak{C}^{*}$ be constructed for $m^{\prime}, n^{\prime}, F_{k}^{\prime}$ as above. Define

$$
\mathfrak{e}_{1}=\mathfrak{e}^{*} \cup\left\{A_{n+1}\right\} \text {. }
$$

Then $\mathfrak{C}_{1}$ has no proper infinite r.e. subclasses (and this proves (b)) and $U \mathcal{C}_{1} \subseteq\{x \mid x \geqq n\}$. Define

$$
\mathfrak{C}_{2}=\mathfrak{e}_{1} \cup\{\{i\} \mid 0 \leqq i \leqq n-1\}
$$

and $\mathfrak{C}_{2}$ is the required class. For given an infinite r.e. subclass $\mathfrak{C}_{3}$ of $\mathfrak{C}_{2}, \mathfrak{C}_{3}-\{\{i\} \mid 0 \leqq i \leqq n-1\}$ is an infinite r.e. subclass of $\mathfrak{e}_{1}$ and therefore is $\mathbb{e}_{1}$. On the other hand, any combination of the $\{i\}$ can be added to $\mathfrak{C}_{1}$.

\section{REFERENCE}

1. P. R. Young, $A$ theorem on recursively enumerable classes and splinters, Proc. Amer. Math. Soc. 17 (1966), 1050-1056.

SiMON Fraser UNIVERSITY 